\title{
Size of Components of a Cube Coloring
}

\author{
Marsel Matdinov
}

Received: 2 May 2012 / Revised: 15 March 2013 / Accepted: 22 April 2013 /

Published online: 10 May 2013

(C) Springer Science+Business Media New York 2013

Abstract Suppose a $d$-dimensional lattice cube of size $n^{d}$ is colored in several colors
so that no face of its triangulation (subdivision of the standard partition into $n^{d}$ small
cubes) is colored in $m+2$ colors. Then one color is used at least $f(d, m) n^{d-m}$ times.

Keywords Cube $\cdot$ Partition $\cdot$ Lattice $\cdot$ Skeleton $\cdot$ Triangulation

Mathematics Subject Classification (1991) $\quad 05 \mathrm{C} 15 \cdot 54 \mathrm{~F} 45$

\section{Introduction}

A theorem attributed to Lebesgue asserts that if a lattice cube of dimension $d$ is colored in $d$ colors then one of the colors has a connected component spanning two opposite facets of the cube. By the standard reasoning with nerves of coverings this means that the covering dimension of the $d$-dimensional cube is at least $d$. The reader may find more information on the Lebesgue theorem and the related notion of Gromov's waist in the remarkable survey [3].

There arises the following natural question: What happens if the number of colors is less than $d$ ? In $[1,5]$ it was conjectured that the size of a monochromatic connected component has a lower bound of order $n^{d-m}$ when $m+1$ colors are used. For $m=d-1$ this follows from the Lebesgue theorem and for $m=1$ this conjecture is proved in [5].

Here we prove this conjecture in a slightly stronger form:

M. Matdinov ( $\varangle)$

Faculty of Mathematics, Higher School of Economics, 7 Vavilova Str., Moscow117312, Russia

e-mail: marmarselsel@mail.ru 
Theorem 1.1 Let a d-dimensional cube $Q$ be partitioned into $n^{d}$ small cubes in the standard way and then the $(m+1)$-dimensional skeleton $Q_{m}$ of this partition is subdivided to the triangulation T. Suppose the vertices of $T$ (equivalently, vertices of $\left.Q_{m}\right)$ are colored in several colors so that no $(m+1)$-face $\sigma \in T$ is colored in $m+2$ different colors. Then one of the colors is used on at least $f(d, m) n^{d-m}$ vertices of $T$.

Remark 1.2 It is also sufficient to assume that every cubic face of $Q_{m}$ of dimension $m+1$ has at most $m+1$ colors on its vertices. Such a point of view allows not to use any triangulation $T$ in the statement of the theorem.

Remark 1.3 If a color $c$ has several connected components then every component can be assumed to be a separate color. So we obtain a monochromatic connected set of at least $f(d, m) n^{d-m}$ vertices of $T$. By adjacent vertices we mean two vertices in a single $(m+1)$-face of $T$. This remark also remains valid if we define adjacent vertices as contained in a single cubic $(m+1)$-face of $Q_{m}$.

A similar theorem was proved independently in [4]:

Theorem 1.4 ([4]) Let the cube $Q$ be partitioned into $n^{d}$ small cubes in the standard way and let every vertex be colored in one of $m+1$ colors. Call a pair of vertices adjacent if they are vertices of a single small cube. Then there exists a monochromatic connected set of vertices of cardinality at least $f(d, m) n^{d-m}$.

This theorem follows from Theorem 1.1 if we color every monochromatic connected component in a separate color. Then, for every small cube $q$ its vertices are colored in at most $m+1$ colors, and therefore the vertices of any $(m+1)$-face of a triangulation of the $(m+1)$-skeleton are colored in at most $m+1$ colors. Thus the new coloring satisfies the assumption of Theorem 1.1.

Remark 1.5 We do not establish any explicit values for $f(d, m)$. The reader may consult the paper [4] for this information.

In Sect. 5 we also give some corollaries of Theorem 1.1 about coverings of a cube or a torus.

\section{Definitions}

We are going to consider the cubic partition $Q_{m}$ (the $m$-skeleton of the full cubic partition of $Q$ ) together with its further triangulation $T$, and define the following chain complexes: The group of $k$-chains $C_{k}(X)\left(X=T, Q_{m}\right)$ is the free abelian group generated by $k$-faces of $T$ (or cubic $k$-faces of $Q_{m}$ ), every face is considered to be oriented in either way. The group of $k$-cochains $C^{k}(X)$ is the group of all group homomorphisms from $C_{k}(X)$ to $\mathbb{Z}$. The boundary operator $\partial_{k}: C_{k} \rightarrow C_{k-1}$ maps every $k$-face (cubic $k$-face) to the sum of its codimension 1 boundary faces with the signs arising from the induced orientation, and is extended linearly to all chains in $C_{k}$. The coboundary operator $\delta_{k}: C^{k} \rightarrow C^{k+1}$ is defined by the formula $\delta_{k}(a)(b)=$ $a\left(\partial_{k+1} b\right)$, where $a \in C^{k}(X), b \in C_{k+1}(X)$. A cycle is a chain with zero boundary; a cocycle is a cochain with zero coboundary. 
Let $C=\left\{c_{0}, \ldots, c_{k}\right\}$ be an ordered set of $k+1$ colors. For every oriented $k$-face of $T$, we assign +1 if its vertices are colored with the colors of $C$ in accordance with the orientation, -1 if the vertices are colored with the colors of $C$ with opposite orientation, and 0 if the face is colored with other colors or some of the colors is used more than once. Thus we define a cochain $\chi(C) \in C^{k}(T)$ and observe the coboundary formula:

$$
\delta \chi(C)=\sum_{c_{k+1}} \chi_{C c_{k+1}},
$$

where we sum over all the colors and by $C c_{k+1}$ we mean the concatenation of $C$ and the new color $c_{k+1}$. If $c_{k+1}$ coincides with a color in $C$ then $\chi_{C c_{k+1}}$ is assumed to be zero. Indeed, the LHS and RHS of (2.1) are $k+1$-cochains, then it is sufficient to test the equality on the generators of $C_{k+1}$, that is on $(k+1)$-faces. This is done immediately by classifying the $(k+1)$-faces into (a) colored in $k+2$ distinct colors containing all the colors of $C$, (b) colored in $k+2$ distinct colors, containing not all the colors of $\mathrm{C}$, and (c) having a pair of vertices colored in the same color.

\section{Plan of the Proof}

The proof will consist in mapping the set of vertices (of the cubic partition) of some $(d-m)$-dimensional big face of $Q$ (there are approximately $n^{d-m}$ vertices) to a set of vertices of $Q$ having the same color, so that the preimage of every vertex has cardinality bounded in terms of $d$ and $m$. This map will be constructed after proving by induction a somewhat tricker statement. Evidently, such a map gives the required result. Possibly, this kind of argument is a new approach to Gromov's waist, compare [3].

In the rest of the proof we consider $(k+1)$-dimensional cubic subcomplexes $Q_{k} \subseteq$ $Q_{m}$, which are $(k+1)$-dimensional cubic skeleta of some $(d-m+k)$-dimensional faces (big faces, not faces of a partition) of the cube $Q$, for $k=m, m-1, \ldots, 0$. In other words, we consider a flag of cubes $Q_{0} \subseteq Q_{1} \subseteq \cdots \subseteq Q_{m}$ such that $Q_{i}$ is a big facet of $Q_{i+1}$, and every time we consider the groups of chains and cochains restricted to $Q_{i}$, etc.

In order to apply simplicial cochains to cubic chains we introduce the function $L: C_{k}\left(Q_{m}\right) \rightarrow C_{k}(T)$ that assigns to any $k$-face $\tau \in Q_{m}$ the sum of simplicial $k$-faces of $T$ that partition $\tau$ with appropriate orientations. Obviously $L$ commutes with the boundary map $\partial$.

We are going to balance the complexes $Q_{k}$ as follows:

Definition 3.1 For every $k$-face $\sigma \in Q_{k}$ we will assign a $(k+1)$-chain $B(\sigma) \in$ $C_{k+1}(T)$ so that for any $(k+1)$-face $\tau \in Q_{k}$ and a set $C$ of $k+1$ colors the following holds:

$$
\chi_{C}(L(\partial \tau)+\partial B(\partial \tau))=0
$$

where we assume that $B$ is linearly extended to $k$-chains of $Q_{k}$. 
We also put $A(\sigma)=L(\sigma)+\partial B(\sigma)$ and use (3.1) in the following form:

$$
\chi_{C}(A(\partial \tau))=0
$$

Let us check that the $(m+1)$-skeleton $Q_{m}$ of $Q$ is already balanced (so that we may put $B(\cdot)=0)$. Let $|C|=m+1$, then

$$
\chi_{C}(L(\partial \tau))=\left(\delta \chi_{C}, L(\tau)\right)=0
$$

since $\delta \chi_{C}=0$ by the formula (2.1) and the assumption of the theorem (no face of $T$ is colored in $m+2$ different colors).

The plan of the remaining part of the proof is following:

- Denote the $k$-skeleton of any facet of the cube corresponding to $Q_{k}$ by $Q_{k-1}$;

- Balance $Q_{k-1}$ by defining a suitable $B: C_{k-1}\left(Q_{k-1}\right) \rightarrow C_{k}(T)$;

- Make sure that in all the expressions $B(\sigma)$ for all $(k-1)$-faces $\sigma \in Q_{k-1}$ every $k$-face $\beta \in T$ is used at most $C(d, k-1)$ times (counted with its multiplicity in the chains $B(\sigma))$.

If this plan passes then on the last stage we have a 1-dimensional skeleton of a $(d-m)$-dimensional cube (containing $n^{d-m}$ small cubes) $Q_{0}$. To every vertex $v \in Q_{0}$ we assign a chain of 1 -faces of $T$ denoted by $B(v)$. Then 0 -chains $A(v)$ are simply sets of vertices of $T$ with integer multiplicities such that the sum of coefficients in every $A(v)$ is 1 . For any color $c$ by (3.2) we obtain that $\chi_{c}\left(A\left(v_{1}\right)\right)=\chi_{c}\left(A\left(v_{2}\right)\right)$ for any pair of adjacent vertices $v_{1}$ and $v_{2}$. Since $Q_{0}$ is a connected graph we obtain that the number $\chi_{c}(A(v))=x_{c}$ does not depend on $v$. The sum over all colors is

$$
\sum \chi_{c}(A(v))=(1, A(v))=1
$$

so there exists a color $c$ with nonzero $x_{c}$. Hence this color is used in every support of the 0-cycle $A(v)$ for every $v$. We have at least $n^{d-m}$ different choices of $v$ and any point colored in $c$ is counted at most $C^{\prime} C(d, 0)$ times (here $C^{\prime}$ is the maximal number of 1 -faces incident to a vertex in $T$ ).

\section{Proof of Theorem 1.1}

So it remains to pass from the balancing of $Q_{k}$ to the balancing of $Q_{k-1}$. Note that for every $k$-face $\tau \in Q_{k-1}$ we have to satisfy the equality (since $\partial^{2}=0$ and $\partial A(\tau)=$ $\partial L(\tau)=L(\partial \tau))$ :

$$
\chi_{C}(\partial A(\tau)+\partial B(\partial \tau))=\chi_{C}(L(\partial \tau)+\partial B(\partial \tau))=0 .
$$

In this formula $A(\tau)$ is already defined, and $B$ is to be defined on $(k-1)$-faces of $Q_{k-1}$. The equality (4.1) follows from the equality:

$$
\chi_{D}(A(\tau)+B(\partial \tau))=0
$$


for every $k$-face $\tau \in Q_{k-1}$ and every set $D$ of $k+1$ colors. Indeed, using (2.1) from (4.2) we obtain

$$
\begin{aligned}
\chi_{C}(\partial A(\tau)+\partial B(\partial \tau)) & =\left(\delta \chi_{C}, A(\tau)+B(\partial \tau)\right) \\
& =\left(\sum_{c_{k}} \chi_{C c_{k}}, A(\tau)+B(\partial \tau)\right)=0 .
\end{aligned}
$$

Now we fix a set $D$ of $k+1$ colors. Define by

$$
\xi_{D}(\tau)=-\chi_{D}(A(\tau))
$$

a $k$-cocycle on $Q_{k}$ since for every $(k+1)$-face $\rho \in Q_{k}$ we have

$$
\xi_{D}(\partial \rho)=-\chi_{D}(L(\partial \rho)+\partial B \partial \rho)=0
$$

because $Q_{k}$ is balanced. To make this cocycle zero (as required in (4.2)) we have to assign to some $(k-1)$-faces $\sigma \in Q_{k-1}$ as $B(\sigma)$ some sets (with coefficients) of $k$-faces $\tau \in T$. Obviously, it suffices to use only those $k$-faces $\tau \in T$ that are colored exactly in the colors of $D$.

The map $\sigma \mapsto \xi_{D}(B(\sigma))$ is going to be a $(k-1)$-dimensional cochain in $C^{k-1}\left(Q_{k-1}\right)$, denote it by $H$. Let us show that if the coboundary of $H$ is $\left.\xi_{D}\right|_{Q_{k-1}}$, then the condition for $D$ holds:

$$
\chi_{D}(A(\tau)+B(\partial \tau))=-\xi_{D}(\tau)+H(\partial \tau)=-H(\partial \tau)+H(\partial \tau)=0
$$

In order to use any $k$-face (out of those colored in $D$ ) at most $C(d, k-1)$ times we have to check that the ratio between the norm (sum of absolute values) of some $(k-1)$ cochain $\eta \in C^{k-1}\left(Q_{k-1}\right)$ such that $\delta \eta=\xi_{D}$ on $Q_{k-1}$ and the number of $k$-faces usable in $B(\sigma)$ (that is, colored in $D)$ is bounded by a constant $C(d, k-1)$.

Let the norm of $\xi_{D}$ as an element of $C^{k}\left(Q_{k}\right)$ equal $M$. By the assumption that in every $B(\tau)$ a $(k+1)$-face of $T$ is used at most $C(d, k)$ times we conclude that every $k$-face of $T$ is used in all $A(\tau)$ at most $C(d, k) C^{\prime}(k)$ times, where $C^{\prime}(k)$ is the maximal number of $(k+1)$-faces containing a given $k$-face of $T$ (it can be bounded independently on the choice of a particular triangulation $T)$. By the formula $\xi_{D}(\tau)=-\chi_{D}(A(\tau))$ we conclude that among the $k$-faces of $T$ there do exist at least $\frac{M}{C(d, k) C^{\prime}(k)}$ "candidates" for $B(\sigma)$. Now it suffices to solve the equation $\delta \eta=\xi_{D}$ on cochains on $Q_{k-1}$ so that the norm $|\eta|$ is at most $C^{\prime \prime}(d, k-1) M$. After that we can assign to cubic faces of $Q_{k-1}$ on which $\eta$ is nonzero several faces of $T$ on which $\xi_{D}$ is nonzero.

Note that $\left|\xi_{D}\right|=M$ and for some codimension 1 cubic section $Q^{\prime}$ of $Q_{k}$ (parallel to $Q_{k-1}$ ) we have $\left|\xi_{D}\right| Q^{\prime} \mid \leq M / n$. Then we use the "filling inequality" (see for example [2], where filling inequalities are widely used):

Lemma 4.1 For a k-dimensional cocycle $\alpha$ on the cubic partition of the d'-dimensional cube $Q^{\prime}$ (in terms of this proof) there exists a $(k-1)$-dimensional cubic cochain $\beta$ such that $\delta \beta=\alpha$ and $|\beta| \leq C_{F}\left(d^{\prime}, k\right) n|\alpha|$. 
By this lemma we select a $(k-1)$-dimensional cochain $\beta$ on $Q^{\prime}$ with norm at most $M C_{F}(d-m+k, k)$ with coboundary $\left.\xi_{D}\right|_{Q^{\prime}}$. Denote the part of $\xi_{D}$ between $Q^{\prime}$ and $Q_{k-1}$ by $\xi_{D}^{\prime}$; this is a cochain with norm at most $M$. As the required $(k-1)$-dimensional cochain $\eta$ on $Q_{k-1}$ we may take

$$
\eta=\beta+\pi_{*}\left(\xi_{D}^{\prime}\right)
$$

with norm at most $\left(C_{F}(d-m+k, k)+1\right) M$. Here $\beta$ is moved from $Q^{\prime}$ to $Q_{k-1}$ by the translation and by $\pi_{*}\left(\xi_{D}^{\prime}\right)$ we mean the direct image under the projection onto $Q_{k-1}$ that drops the dimension by 1 . The cochain $\pi_{*}\left(\xi_{D}^{\prime}\right)$ can be defined explicitly (thanks to the cubic complexes that we use) as taking any ( $k-1)$-face $\sigma \in Q_{k-1}$ to the sum of values (with appropriate signs) of $\xi_{D}^{\prime}$ on $k$-faces of $Q_{k}$ that project onto $\sigma$. In other words

$$
\pi_{*}\left(\xi_{D}^{\prime}\right)(\tau)=\xi_{D}^{\prime}\left(\pi^{-1}(\tau)\right)
$$

So we satisfy the equality (4.2) for a particular color set $D$. Now we can add the chains $B(\sigma)$ corresponding to different sets $D$. The expressions $B(\sigma)$ for every particular $D$ contained exclusively $k$-faces colored in the colors in $D$. Hence for different $D$ we use different faces, which guarantees the bounded multiplicity of the union of all $B(\sigma)$. The faces of $B(\sigma)$ colored in $D$ do not affect the equality (4.2) for another set $D^{\prime}$ (not obtained from $D$ by a permutation). Now to complete the proof it remains to prove the lemma.

Proof of Lemma 4.1 The proof is similar to the proof of [4, Lemma 2.6], which is stated in terms of cycles Poincaré dual to the cocyles in this proof.

Put $\alpha_{0}=\alpha$. We are going to build a cocycle $\alpha_{i+1}$ out of $\alpha_{i}$ as follows. Take a hyperplane section $Z$ of $Q^{\prime}$ parallel to a pair of its opposite facets so that $\left|\alpha_{i}\right|_{Z} \mid \leq$ $\left|\alpha_{i}\right| / n$. This is possible by the Dirichlet principle.

Define a $(k-1)$-cochain $\beta_{i}$ as follows:

$$
\beta_{i}(\tau)=\alpha_{i}\left(\left[\tau, \pi_{Z}(\tau)\right]\right)
$$

where $\left[\tau, \pi_{Z}(\tau)\right]$ is (at most $k$ )-dimensional parallelepiped between $\tau$ and its projection onto $Z$ with appropriate sign.

Now we put

$$
\alpha_{i+1}=\alpha_{i}-\delta \beta_{i}
$$

and note that $\left|\beta_{i}\right|$ is at most $n\left|\alpha_{i}\right|$. Note also that $\alpha_{i+1}$ takes the same values as the translation of $\left.\alpha_{i}\right|_{Z}$ on sections parallel to $Z$ and is zero on any face orthogonal to $Z$.

After several such operations for different directions of $Z\left(d^{\prime}-k+1\right.$ will be enough) the cocycle $\alpha_{i+1}$ becomes zero. We have the inequality:

$$
\left|\alpha_{i+1}\right| \leq\left|\alpha_{i}\right| \leq \cdots \leq|\alpha|
$$


If we take $\beta=\sum_{i} \beta_{i}$ then the required inequality holds with constant $C_{F}\left(d^{\prime}, k\right) \leq$ $d^{\prime}-k+1$.

\section{Some Corollaries}

We give some topological corollaries of Theorem 1.1:

Corollary $5.1{ }^{1}$ Let a d-dimensional cube $Q$ be covered by a finite family of closed sets $C_{i}$ so that no point is covered more than $m+1$ times. Then one of the sets $C_{i}$ intersects at least $d-m$ pairs of opposite facets of $Q$.

Remark 5.2 This is a generalization of the Lebesgue theorem.

Proof Let us make a corresponding color for every covering set. Let $r$ be a positive distance and $x$ be a point in $Q$. Let $u(r, x)$ be the number of the covering sets intersecting the closed ball of radius $r$ centered at $x$. Put $f(x)=\sup \{r: u(r, x) \leq m+1\}$, which may attain $+\infty$ value.

Consider a point $x \in Q$. For every $C_{i}$ not containing $x$ there exists a neighborhood of $x$ in $Q$ disjoint from $C_{i}$ (since $C_{i}$ is closed). The intersection of the finite number of such neighborhoods makes a neighborhood of $x$ intersecting at most $m+1$ sets of $C_{i}$. Hence $f(x)$ is positive on $Q$. Moreover, if $f(x)=r>0$, then $f(y) \geq r-|x-y|$, because the ball of radius $r-|x-y|$ centered at $y$ is contained in the ball of radius $r$ centered at $x$. Therefore $f$ is lower semicontinuous on the compactum $Q$ and therefore must attain its positive minimum somewhere on $Q$. Let $R>0$ be this minimum.

The definition of $R$ means that any open $R$-neighborhood of any $x \in Q$ intersects at most $m+1$ sets of the covering. Now we partition the cube $Q$ into $n^{d}$ small cubes in the standard way and take $n$ so large that every side of a small cube has length less than $R / d$. If we color every vertex of the cubic partition into the color, corresponding to any covering set that contains this vertex, then we obtain a coloring which uses at most $m+1$ colors on the vertices of any small cube.

Let us include $Q$ into the cube $2 Q$ of size $(2 n)^{d}$ and repeat the coloring of $Q$ using reflections with respect to the halving hyperplanes of $2 Q$. Then we extend the coloring onto the whole $\mathbb{Z}^{d}$ with translations by $\pm 2 n$ along the coordinate axes. Let us see what happens with a color $c_{i}$. Following the discussion in the introduction (the comparison with Karasev's theorem) we assume that the color $c_{i}$ makes a connected subset of $Q$. The vertices of $\mathbb{Z}^{d}$ colored in $c_{i}$ can be decomposed into connected components; denote one of them by $c_{i}^{\prime}$. If the component $c_{i}$ spans a pair of opposite facets (orthogonal to a base vector $e_{j}$ ) in $Q$ then $c_{i}^{\prime}$ is invariant under the translation by $\pm 2 n e_{j}$. Otherwise $c_{i}$ does not touch one of the facets orthogonal to $e_{j}$ and $c_{i}^{\prime}$ is trapped between a pair of hyperplanes orthogonal to $e_{j}$ at distance $2 n$ from each other.

So the free Abelian group $\Lambda_{i}$ of translational symmetries of $c_{i}^{\prime}$ has dimension exactly $\ell$, where $\ell$ is the number of pairs of opposite facets of $Q$ intersected by $c_{i}$ and $c_{i}^{\prime}$ can be obtained from $2 Q \cap c_{i}^{\prime}$ by translations in $\Lambda_{i}$. If we intersect $c_{i}^{\prime}$ with a large cube $Q^{\prime}$ of size $(2 n N)^{d}$ then the cardinality of $c_{i}^{\prime} \cap Q^{\prime}$ has the growth order $N^{\ell}$ for

\footnotetext{
1 The statement of this corollary is suggested by R. Karasev as a simpler version of Corollary 5.3.
} 
varying $N$. By Theorem 1.1 some $c_{i}^{\prime} \cap Q^{\prime}$ must have the number of vertices of order at least $N^{d-m}$; so for some of $c_{i}^{\prime}$ we must have $\ell \geq d-m$.

Corollary 5.3 Let a d-dimensional torus $T^{d}$ be covered by open sets $C_{i}$ so that no point is covered more than $m+1$ times. Then for some $C_{i}$ the image of $H_{1}\left(C_{i}\right)$ in $H_{1}\left(T^{d}\right)=\mathbb{Z}^{d}$ has dimension at least $d-m$.

Remark 5.4 The sets have to be open so that the connectedness and the arcwise connectedness coincide.

Proof As in the previous proof we pass from the covering of $T^{d}$ to a fine enough triangulation of a covering cube $Q$, which subdivides the cubic partition into $n^{d}$ small cubes. Then open covering can be refined to a closed covering by the paracompactness of $Q$. Then we assume that the vertices of the triangulation are colored so that no face has more than $m+1$ colors. Duplicating $Q$ by translations we obtain a large cube $Q_{N}$ with side length $N n$ and the corresponding coloring. By gluing the opposite facets of $Q_{N}$ we obtain a torus naturally $N^{d}$-fold covering $T^{d}$.

By Theorem 1.1 in $Q_{N}$ we have a monochromatic connected component $S$ with size of order $N^{d-m}$. Let $S_{1}$ be the maximal intersection of $S$ with a residue class modulo $Q$ (points are equal modulo $Q$ if the differences of their coordinates are divisible by $n$ ). Then $\left|S_{1}\right| \geq|S| / n^{d} \geq \frac{N^{d-m}}{n^{d}}$.

Note that a projection of a monochromatic path in $S$ starting in a point of $S_{1}$ with coordinates $\left(x_{1}, \ldots, x_{d}\right)$ and ending in a point of $S_{1}$ with coordinates $\left(y_{1}, \ldots, y_{d}\right)$ is a monochromatic closed loop in $T^{d}$ representing the homology class $\left(\frac{y_{i}-x_{i}}{n}\right)_{i}$. So it suffices to show that the dimension of the linear space generated by pairwise differences of $S_{1}$ is at least $d-m$ (the covering set corresponding to $S$ will be the one required). Equivalently, we have to show that the dimension of the affine hull of $S_{1}$ is at least $d-m$.

Assume the contrary: The dimension of the affine hull of $S_{1}$ is at most $d-m-1$. Then $S_{1}$ is contained in at most $(d-m-1)$-dimensional affine subspace $L$ and the number of vertices in $Q_{N} \cap L$ (and therefore the number of vertices in $S_{1}$ ) is at most $(N n)^{d-m-1}$. For large enough $N$ we obtain a contradiction with the inequality $\left|S_{1}\right| \geq \frac{N^{d-m}}{n^{d}}$.

Acknowledgments The author thanks Alexey Belov-Kanel for drawing attention to this problem and numerous discussions, Roman Karasev for his help in writing the text and translating it to English, and the referee for numerous useful remarks and suggestions. This research was supported by the President's of Russian Federation Grant MD-352.2012.1 and the Federal Program "Scientific and scientific-pedagogical staff of innovative Russia” 2009-2013

\section{References}

1. Belov-Kanel, A., Ivanov-Pogodaev, I., Malistov, A., Kharitonov, M.: Colorings and clusters. In: 22nd Summer Conference International Mathematical Tournament of Towns, Teberda, Karachai-Cherkess, 02-10 August 2010. http://olympiads.mccme.ru/lktg/2010/2/2-1en.pdf

2. Gromov, M.: Singularities, expanders, and topology of maps. Part 2: from combinatorics to topology via algebraic isoperimetry. Geom. Funct. Anal. 20(2), 416-526 (2010)

3. Guth, L.: The waist inequality in Gromov's work. http://math.mit.edu/ guth/Exposition/waist.pdf 
4. Karasev, R.N.: An analogue of Gromov's waist theorem for coloring the cube. Discrete Comput. Geom. 49(3), 444-453 (2013). doi:10.1007/s00454-013-9490-4

5. Matoušek, J., Přívětivý, A.: Large monochromatic components in two-colored grids. SIAM J. Discret. Math. 22(1), 259-311 (2008) 